\section{$\Omega$}

Renata L. Riha

(1]

Dept of Sleep Medicine, Royal Infirmary of Edinburgh, Edinburgh, UK.

\title{
Breathe: respiratory health and disability
}

Welcome to the December issue of Breathe! We hope that over the last year you have enjoyed the varied content and selection of topics addressed in this journal, and found at least some sufficiently novel to take time out of your busy schedules to read.

This issue is dedicated to respiratory health and disease in the context of disability, both in adults and children. We are privileged to have contributions from experts in the field covering a spectrum of respiratory issues from the use of inhalers for asthma in patients with intellectual disability to the prevalence, impact and management of sleep disordered breathing in adults with spinal cord injury.

As an inclusive society, we cater to patients of all ages, abilities and backgrounds, yet most of the respiratory textbooks we first learn from focus on the physiology of the "ideal 70-kg man" or the monosymptomatic patient without other comorbidities or disabilities, which can affect everything from disease expression to treatment choices.

In this issue, we have eschewed the Physiology Masterclass for a much longer paper focussing on respiratory physiology in patients with neuromuscular disorders. We also have a case study highlighting the importance of recognising sleep disordered breathing in patients with cerebral palsy

Additional contributions appear as online exclusives. Please, do come back to us to read and share this material (breathe.ersjournals.com) as it contains essential insights into very poorly studied but very important areas of lung and sleep health that are seldom covered in standard medical literature and training.

We also urge you to participate in our online journal club, the inaugural edition of which is taking place on Tuesday, December 13, 2016. The journal club will be chaired by Frits Franssen (Secretary of the ERS Rehabilitation and Chronic Care Group (1.2)). The paper to be discussed ties in with the general theme of this issue [1] and can be downloaded from doi.org/10.1016/S14744422(16)30233-2 The author of this paper will be available as a discussant, and we hope that you will be able to participate with your own questions and comments.

We hope the journal club will become a regular feature for the ERS and look forward to your collaboration in making this a success. More details and the registration form are available at https://goo.gl/forms/RsZaDkIMRCUeAcTe2

As always, my thanks go to all the contributors to this issue, the tireless efforts and patience of the editorial team, and ongoing support by you, our readers, in making this journal a success. Wishing you a peaceful and safe festive season, and happy reading!
Cite as: Riha RL. Breathe: respiratory health and disability. Breathe 2016; 12: 303-304 


\section{Conflict of interest}

None declared.

Reference

1. Gonzalez-Bermejo J, Morélot-Panzini C, Tanguy ML, et al. Early diaphragm pacing in patients with amyotrophic lateral sclerosis (RespiStimALS): a randomised controlled triple-blind trial. Lancet Respir Med 2016; 15: 1217-1227. 